Concerning the toxicity of acetanilid and bicarbonate combinations for muscle-nerve preparations.

\title{
By David I. Machi.
}

Pharmological Laboratory, Johns Hopkins University.]

It is well known, through the work of Worth Hale, ${ }^{1}$ that acetanilid when given in combination with small doses of sodium bicarbonate, is less toxic than when administered in the same doses alone. The studies of Macht, Greenberg and Isaacs on the influence of antipyretics on the acuity of hearing ${ }^{2}$ have also shown an interesting difference between the effects of acetanilid when given alone and acetanilid when combined with sodium bicarbonate. The explanations given for the above peculiar synergism are most unsatisfactory. In connection with a pharmacological study of various antipyretics, the author investigated the effect of acetanilid solutions on muscle-nerve preparations which may throw some light upon the above-mentioned phenomena.

Two gastrocnemius muscle-nerve preparations of a frog are immersed simultaneously in physiological sodium chloride solution and the limits of both the muscle and nerve excitability to the electric shocks of an induction coil are determined. One of the preparations is then immersed in a solution of acetanilid in the same saline solution, and the other preparation is immersed in a solution of acetanilid of exactly the same strength but containing some sodium bicarbonate (I-I000 or even weaker). On testing the excitability of the muscles and nerves at regular intervals after treatment with the drugs, it was found that the plain acetanilid solution tends to paralyze and finally kill the sciatic nerve more quickly than the solution of acetanilid plus bicarbonate. The following protocol of an experiment thus performed will serve as an illustration. The slower toxic action of the acetanilid and bicarbonate combination, all other factors being equal would, it is fair to assume, render the drug less poisonous when injected into an

${ }^{1}$ U. S. Hygienic Lab. Bulletin No. 53 (1909).

2 Proc. Soc. for EXrer. BIOL. ANd MED., I9Ig, xvii, 22, 23. 
animal than acetanilid would be if injected alone, for in case of the more slowly acting combination the animal has a longer period of time for the excretion of the poison. The author does not at all presume to offer these experiments as a complete explanation of the above-described synergism, but it is thought that the present findings are at least a little tangible contribution towards the explanation of the peculiar phenomenon.

EXPERIMENT, OCTOBER 28, 1919.

Stimulation of Gastrocnemii Muscles of Rana Clamata.

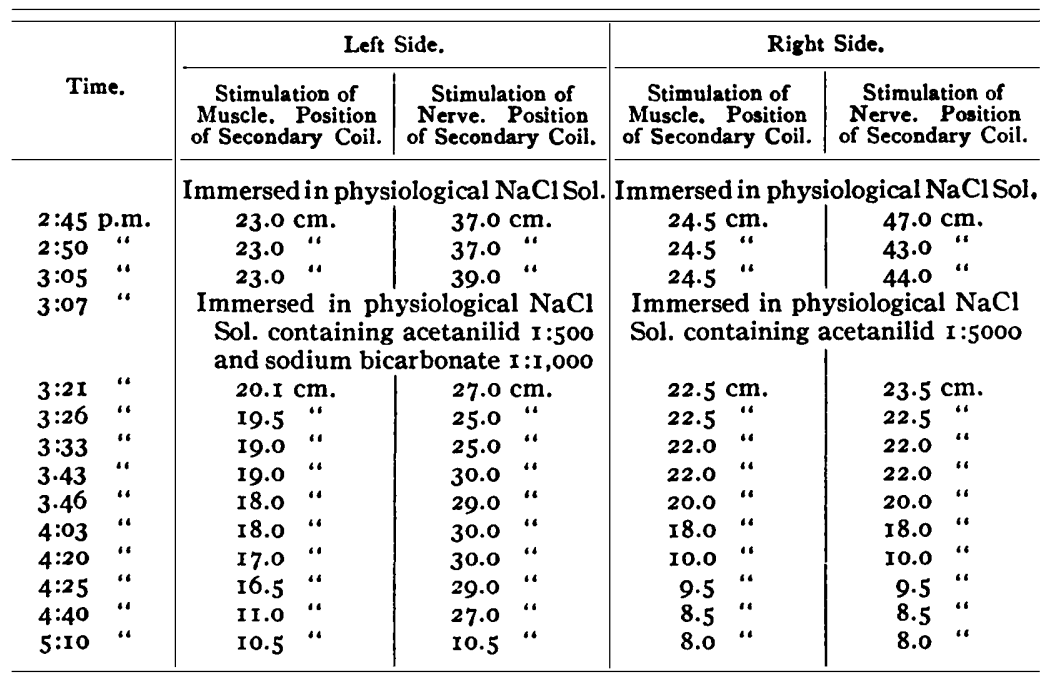

\section{5 (1485)}

A note on the carbohydrates of the root of the cat-tail (Typha latifolia).

By Zalia Jencks (by invitation).

[From the Sheffield Laboratory of Physiological Chemistry, Yale University, New Haven, Conn.]

In a recent communication ${ }^{1}$ the root of the cat-tail has been recommended as a valuable food product for man. An analysis is recorded to indicate that the material contains $8 \mathrm{I}$ per cent. of carbohydrates. No evidence is presented however, as to the

1 P. W. Claasen, The Scientific Monthly, I919, Vol. 9. No. 2. 